\title{
PERSPECTIVES IN DIAGNOSIS AND THERAPEUTICS IN HEMATOLOGY
}

\author{
Guillermo José Ruiz-Argüelles ${ }^{1 *}$ AND David Gómez-Almaguer ${ }^{2}$ \\ ${ }^{1}$ Clínica Ruiz de Puebla, Centro de Hematología y Medicina Interna, Puebla; ${ }^{2}$ Hospital Universitario, \\ Universidad Autónoma de Nuevo León, Monterrey, Mexico
}

\begin{abstract}
In this article, the perspectives on both the diagnosis and treatment of acute leukemias, chronic leukemias, multiple myeloma, anemia, platelet, and coagulation disorders are briefly discussed. We emphasize the limitations facing the practice of hematology in low- and middle-income countries. (REV INVEST CLIN. 2021;73(5):306-9)
\end{abstract}

Key words: Myeloma. Leukemia. Benign hematological diseases. Anemia. Platelet diseases. Coagulation.

The worldwide perspective of both the diagnosis and treatment of hematological diseases is vast and promising, but unfortunately, hampered by the rising costs of both the diagnostic studies and the novel therapeutic drugs. As a result, the gap between diagnostic and therapeutic options for persons living in low- and middle-income countries (LMIC) and those from high-income countries (HIC) will sadly increase, thus limiting the benefits to most persons living in LMIC, where more than $80 \%$ of the world's inhabitants dwell ${ }^{1}$. Physicians will become more acquainted with the financial toxicity of many of the novel treatments of hematological diseases. Drug companies will continue to select key opinion leaders (KOL) and paid lecturers to disseminate knowledge of the treatment of these diseases based mainly on economic reasons, rather than on the comprehensive well-being of persons $^{1,2}$. The median age of the paid lecturers or KOL will decrease substantially, whereas the number of virtual conferences, seminars, and meetings will greatly increase.

Although there are currently several worldwide perspectives in both diagnosis and treatment of hematological malignancies, due to space constrains, we will only briefly discuss those in acute leukemias (AL), chronic leukemias, and plasma cell myelomas (PCM) as well as in selected benign hematological diseases, emphasizing the limitations facing the practice of hematology in LMIC.
*Corresponding author:

Guillermo J. Ruiz-Argüelles

E-mail: gruiz1@clinicaruiz.com
Received for publication: 29-05-2021

Approved for publication: 04-06-2021

DOI: 10.24875/RIC.21000292

0034-8376 / @ 2021 Revista de Investigación Clínica. Published by Permanyer. This is an open access article under the CC BY-NC-ND license (http://creativecommons.org/licenses/by-nc-nd/4.0/). 


\section{PERSPECTIVES IN HEMATOLOGICAL MALIGNANCIES}

\section{Acute Leukemias}

Morphology as a tool to make the diagnosis and classification of AL will be substantially less important. Cytomics, flow cytometry, will be routinely employed in hematology laboratories to assess and analyze the expression of cell surface antigens to define the nature of the leukemic cells. The identification of molecular genetic markers of $A L$ such as BCR/ABL, PML/ RARa, AML1/ETO, inv16, FLT-3, NPM-1, and others, currently done by molecular biology, will be replaced by next generation sequencing techniques. The therapeutic consequences of the adequate identification of molecular markers of the AL will be more numerous. More specific and less-toxic treatments will be employed, and chemotherapy-free treatments will be more frequently used ${ }^{3}$. The cost of target-specific anti-leukemic treatments such as the tyrosine-kinase inhibitors (TKls), all trans-retinoic acid, arsenic trioxide, midostaurin, venetoclax, and others, currently unaffordable for most persons living in LMIC, will most likely decrease once the generic versions or the imported similars of these drugs become more widely available. Leukemia researchers will have to modify the endpoint of their studies focusing in the overall survival of the patients and their quality of life (QoL). Hematopoietic stem cell transplants (HSCT) will be simplified and conducted in cases in which an affordable target-specific treatment is not available. HSCT from haploidentical donors (parents, sons, daughters, and HLA non-identical siblings) will be done more frequently, replacing the transplants with placental blood ${ }^{4}$. The treatment with chimeric antigen receptor $T$ cells (CAR-T cells) will be more widely available, but its cost will be prohibitive for most patients living in LMIC. Novel methods to offer CAR-T cell treatment will be developed to turn them more affordable for persons living in underprivileged circumstances.

\section{Chronic leukemias}

As in AL, morphology will become less critical in the study and treatment of persons with chronic leukemias. The identification of the BCR/ABL fusion transcript will be a routine practice in the assessment of persons with chronic myelogenous leukemia (CML). Imatinib will be less employed as the initial treatment of these persons since there are now generic versions of it, and the drug companies will push the use of other TKIs as initial treatment, such as dasatinib, nilotinib, and ponatinib. HSCT will be less employed in the treatment of persons with CML, mainly in HIC. The follow-up of the molecular marker of the disease will be done routinely to define the treatment more precisely.

The use of novel and expensive drugs in the initial treatment of persons with chronic lymphocytic leukemia (CLL), such as ibrutinib, acalabrutinib, venetoclax or obinutuzumab, and others, will be restricted to persons living in $\mathrm{HIC}$, and researchers in LMIC countries will very carefully assess if a molecular remission of the disease is strictly necessary to improve the well-being, the QoL and the long-term survival of patients. As a result of the improved diagnostic capacity of CLL in laboratories, physicians in LMIC will face more cases of CLL in whom specific treatment is not needed at all, but they will also confront the pressure of the drug companies to start novel and expensive treatments aiming for the disappearance of minimal residual disease (MRD).

\section{Plasma Cell Myelomas}

The investigation of free light chains in the serum will become routine practice in the study of persons with low back pain, anemia, renal failure, nephrotic syndrome, and hypercalcemia; general practitioners will become more aware of PCM being proteiform and worth considering its diagnosis in difficult cases. The AL-based sequential phases of induction/consolidation/maintenance in patients with PCM will most likely prevail. It will become clear if the use of 2,3 , or 4 drugs in the induction phase is strictly needed, as well as the deepness of the response before the transplant ${ }^{3}$. It is rather obvious that the number of drugs in the induction phase will be directly related to the cost of the treatment and inversely related to the likelihood of being employed in patients with PCM living in LMIC. Analysis of the long-term overall survival coupled with the assessment of QoL will more clearly define the objective of the treatment of persons with MM and fewer cases of refractory forms of the disease will be found ${ }^{3}$. It will be defined if a stringent remission, with no evidence of MRD, is strictly necessary to improve the overall survival and QoL of the patients. Immunomodulatory drugs such as thalidomide, lenalidomide, and pomalidomide will become 
more affordable as the result of the availability of generic forms. Autologous HSCT will remain the main consolidation tool in PCM, after the induction to remission, and simplified forms of conducting HSCT, mainly on an outpatient basis, will prevail 33,4 . The maintenance phase of the treatment of the disease will remain as mandatory and more cases of MM will survive decades, thus entertaining the concept of "true" cure of the disease. For relapsed or refractory cases, drugs better than daratumumab will be shown to work. Turning CAR-T cells therapy more affordable will make this treatment available to refractory or relapsed myeloma patients.

\section{PERSPECTIVES IN BENIGN HEMATOLOGY}

The so-called benign hematology includes several blood diseases such as anemia, platelet, and coagulation-thrombosis alterations, and leucocyte pathology. At present, much more money from pharmaceutical companies and research groups is devoted to malignant hematology, "- money is where cancer is -;" however, we are facing interesting developments in this field, with better and faster diagnostic laboratory tools and personalized and precise medicine being now a reality.

\section{Laboratory perspectives}

More rapid and precise diagnostic tools will appear soon. Automatization and precision will be the way to go in the diagnosis of many benign hematological diseases. It is still important to combine traditional automated analysis, morphological inspection, and the use of novel techniques such as flow cytometry analysis, advancing imaging processing, genomic testing, and microfluidics. It will not be long before they become of common and everyday use in hematology laboratories. The digital world is already here, and we will be able to observe sophisticated images using the smart phones' cameras, instead of using complex computer software, and microscope cameras. Highquality morphology images will be analyzed in a very practical way.

\section{Anemia}

There are new perspectives in the early intervention in autoimmune hemolytic anemia: Rituximab up-front with corticosteroids is a reality and low dose $(100 \mathrm{mg}$ weekly, 4 weeks) is already useful and soon this should be the guidelines' recommendation5. Other antibodies will emerge, like daratumumab, an antiCD38 antibody indicated in PCM, but with potential therapy for several autoimmune diseases because of its activity against plasma cells.

In the treatment of paroxysmal nocturnal hemoglobinuria (PNH); the use of eculizumab, currently a costly option, is adequate to avoid complement damage to red cells, although the drug must be delivered intravenously every 2 weeks. New molecules with long lasting activity will replace eculizumab, with similar activity and more friendly administration: ravulizumab has been recently FDA approved, the drug being delivered every 8 weeks. Other similar molecules will appear soon. The high cost of this kind of drugs is prohibitive in LMIC, where the only curative option is HSCT; in LMIC, there will be more transplant centers with less-toxic transplantation procedures to treat $\mathrm{PNH}$ patients ${ }^{4}$.

In aplastic anemia (AA); the use of thrombopoietin analogues is already incorporated to the classical combination of thymocyte anti-globulin and cyclosporine; this triple combination is considered ideal if HSCT is not an option. The use of other thrombopoietin analogs will be tested and potentially incorporated in bone marrow failure soon; however, HSCT is still the best treatment option for fit patients 50 years old or younger. The use of haploidentical HSCT is already in the therapeutic armamentarium of $A A$; therefore, this procedure will be more frequently employed in young and fit patients. The number of transplanted patients will increase, and improvements in support therapy will appear making this risky procedure more secure ${ }^{4}$.

In hereditary anemia like thalassemia or sickle cell disease (SCD), we are waiting for treatments with curative potential. In this setting, gene therapy will be a reality soon. Drugs like luspatercept, a recombinant fusion protein that acts as a trap for activin II receptors promoting late-stage erythropoiesis, is already in the market, but with the caveat of its high cost. HSCT also will be more effective, available, and friendly for persons with thalassemia and SCD. Interesting future options such as JAK2 inhibitors, minihepcidins, and ferroportin inhibitors are being studied. 
In the case of SCD, novel options for treatment are directed to modify the genotype, to target the HbS polymerization, or to target vaso-occlusion and inflammation. Several new molecules are already in clinical trials, and the results will be soon available. Gene therapy is one of the most promising therapies and a number of clinical trials based on gene addition using viral vectors to deliver therapeutic genes (antisickling beta-globin) with curative purposes are being conducted. In addition, there are promising gene editing therapies focused on correcting single base mutation in patients with SCD. In these strategies, sophisticated molecular technologies are needed to make changes in genes to modify the mutation, thus inducing the expression of therapeutic proteins ${ }^{6}$.

\section{Platelets and coagulation}

Hemophilia will soon be treated with gene therapy. At present, long-lasting coagulation factors are already making improvements on QoL in such patients. New molecules and antibodies such as emicizumab are coming, and the treatments of inhibitors are being a success.

Primary immune thrombocytopenia (PIT) will be cured immediately after diagnosis. Nowadays, the guidelines recommend the "old fashioned" treatment of corticosteroids and IV immunoglobulin, but the use of combinations of rituximab/corticosteroids, eltrombopag/dexamethasone, mycophenolic acid/dexamethasone is already in study and will eventually be incorporated in the PIT treatment guidelines. New molecules such as fostamatinib and eltrombopag will be more affordable and will also have a role in PIT.
In persons with thrombotic thrombocytopenic purpura (TTP), plasma exchange is still the first-line treatment; however, the new drug caplacizumab has the potential to control TTP without the need for plasma exchange. In some selected patients the use of early rituximab plus corticosteroids has the same potential ${ }^{7}$.

\section{CONCLUSIONS}

The future of both the diagnosis and treatment of hematological diseases is wide and promising, but hampered by their rising costs. New ideas on the ways to diagnose and treat these diseases should make more affordable all these novelties for persons living in LMIC, which currently represent more than $80 \%$ of all the inhabitants of the world.

\section{REFERENCES}

1. Ruiz-Argüelles GJ, Steensma DP. Staunching the rising costs of haematological health care. Lancet Haematol. 2016;3:e455.

2. Ruiz-Argüelles GJ. A new breed in the teaching of medicine: paid lecturers, trainers or speakers. Acta Haematol. 2016; 135:191-2.

3. Ruiz-Argüelles GJ, Gómez-Almaguer D. Lessons learned treating patients with multiple myeloma in resource-constrained settings. Curr Hematol Malig Rep. 2021;16:40-44.

4. Ruiz-Argüelles GJ. Lessons learned starting a bone marrow transplantation programme in a resource-constrained setting. Lancet Haematol. 2020;7:e509-10.

5. Jaime-Pérez JC, Rodríguez-Martínez M, Gómez-de-León A, Tarín-Arzaga L, Gómez-Almaguer D. Current approaches for the treatment of autoimmune hemolytic anemia. Arch Immunol Ther Exper. 2013;61:385-95.

6. Salinas Cisneros G, Thein SL. Recent advances in the treatment of sickle cell disease. Front Physiol. 2020;11:439.

7. Gómez-De León A, Villela-Martínez LM, Yáñez-Reyes JM, Gómez-Almaguer D. Advances in the treatment of thrombotic thrombocytopenic purpura: repurposed drugs and novel agents. Expert Rev Hematol. 2020;13:461-70. 